\title{
Falls after spinal cord injury: a systematic review and meta-analysis of incidence proportion and contributing factors
}

\author{
Alia Khan ${ }^{1} \cdot$ Clara Pujol $^{1} \cdot$ Mark Laylor $\mathbb{1}^{2} \cdot$ Nikola Unic $^{1} \cdot$ Maureen Pakosh $^{3} \cdot$ Jaclyn Dawe $^{1} \cdot$ \\ Kristin E. Musselman $\mathbb{D}^{1,4}$
}

Received: 12 November 2018 / Revised: 11 March 2019 / Accepted: 13 March 2019 / Published online: 9 April 2019

(C) International Spinal Cord Society 2019

\begin{abstract}
Introduction Falls are detrimental to the well-being of individuals with spinal cord injury (SCI). To establish effective fall prevention initiatives, a comprehensive understanding of falls after SCI is needed.

Objectives To report the incidence proportion of falls and summarize the factors contributing to falls in individuals with SCI.

Study design Systematic review and meta-analysis.

Methods Eight databases were searched. Abstracts/full articles were screened by two researchers independently. Data concerning study design, participant characteristics, and the incidence proportion, factors, and consequences of falls were extracted. Risk of bias was assessed using a domain-based approach that considered sampling and measurement bias. The incidence proportions of falls were pooled for ambulators and wheelchair users separately using random-effects metaanalyses, and compared descriptively for inpatients and community-dwelling individuals. Fall-related factors were organized according to the Biological, Behavioral, Social \& Economic and Environmental Model of fall risk.

Results The search resulted in 1706 articles; 24 unique studies were included. The risk of sampling bias was high. All but one study focused on community-dwelling individuals; $78 \%$ (95\% confidence interval $73-83 \%, I^{2}=0 \%$ ) of ambulators and $69 \%$ (95\% confidence interval $60-76 \%, I^{2}=59 \%$ ) of wheelchair users fell $\geq 1$ over 12 months. In contrast, only $13 \%$ of inpatients fell. Most fall-related factors were categorized as biological (e.g., muscle weakness), behavioral (e.g., inattentiveness) or environmental (e.g., uneven surfaces).

Conclusions Falls are frequent among community-dwelling individuals with SCI. A variety of biological, behavioral, and environmental factors contribute to falls, some of which are modifiable and may be addressed through SCI-specific fall prevention initiatives.
\end{abstract}

Supplementary information The online version of this article (https:// doi.org/10.1038/s41393-019-0274-4) contains supplementary material, which is available to authorized users.

Kristin E. Musselman

Kristin.Musselman@uhn.ca

1 SCI Mobility Lab, Toronto Rehabilitation Institute, University Health Network, Toronto, ON, Canada

2 Department of Kinesiology, Faculty of Applied Health Sciences, University of Waterloo, Waterloo, ON, Canada

3 Library \& Information Services, Toronto Rehabilitation Institute, University Health Network, Toronto, ON, Canada

4 Department of Physical Therapy, Faculty of Medicine, University of Toronto, Toronto, ON, Canada

\section{Introduction}

The consequences of falls result in significant individual and societal burden. Fall-related injuries, such as fracture and head injury, cost health-care systems billions of dollars every year [1]. Not only may individuals who fall experience an injury, but they may develop a post-fall syndrome characterized by reduced mobility, depression, decreased autonomy, and reduced participation in daily activities [2]. At present, there is a strong desire among health-care administrators, rehabilitation professionals and researchers to address the "emerging public health crisis" [3] of falls and identify effective initiatives to prevent falls among vulnerable populations, such as those with spinal cord injury (SCI). 
The sensorimotor deficits characteristic of SCI, such as lower limb muscle paresis or paralysis, reduced trunk control, and decreased sensation and proprioception, contribute to impaired balance control. The inability to maintain balance is likely a key underlying cause of falls among individuals with SCI, although it is likely that other intrinsic factors (e.g., spasticity, impulsive behavior) or extrinsic factors (e.g., characteristics of the environment) contribute to falls in this population. Currently, we lack a comprehensive understanding of the frequency of falls among the SCI population and what factors contribute to falls in this group. A synthesis of the fall-related literature in SCI will inform the development of fall prevention initiatives that are tailored to the SCI population. For example, understanding which sub-group of individuals with SCI are at the greatest risk of falling, and which contributing factors are modifiable, will enable the rehabilitation team to focus fall prevention initiatives on areas likely to have the greatest impact.

Compared with other neurological populations, our knowledge of falls and how to prevent them after SCI is lacking [4-7]. Fall prevention interventions may facilitate participation in much-needed recreational, educational, and employment opportunities for the SCI population. Despite being significantly younger than individuals with other neurological conditions, the SCI population has a greater proportion with low education (20\%), and with an annual household income of $<\$ 25,000$ USD (34\%) [8]. Further, the demographics of SCI are changing [9-11]. The age of onset of SCI is increasing and more individuals with SCI are presenting with pre-existing health conditions [9, 10, 12]. Both increasing age and co-morbid conditions are known risk factors for falls [8]. Thus, it is reasonable to expect the frequency of falls in SCI to climb, increasing the need for effective fall prevention strategies.

In order to increase our understanding of falls after SCI, we completed a systematic review and meta-analysis that aimed to: (1) determine the incidence proportion of falls among people with SCI; and (2) identify factors associated with falls in these individuals. The findings will increase our understanding of where further research and monitoring are needed, and will assist with the development and implementation of SCI-specific fall prevention interventions.

\section{Materials and methods}

This systematic review and meta-analysis were conducted in accordance with the Preferred Reporting Items for Systematic Reviews and Meta-Analysis (PRISMA) guidelines [13]. This review had no registered protocol.

\section{Data sources \& search strategy}

A literature review was completed with the help of an information specialist (MP) at the University Health Network, Toronto, Canada. Eight electronic databases were searched from inception to 24 May 2018: Medline (OVID), Cochrane Controlled Trials Register (CCTR), Cochrane Database of Systematic Reviews (COCH), Embase, PsycINFO, PubMed (excluding Medline), Cumulative Index to Nursing and Allied Health Literature (CINAHL), and Physiotherapy Evidence Database (PEDro). The search strategies utilized the PICO framework (population, intervention, comparator, and outcomes), valid subject headings as appropriate for each database, and free text terms relevant to the topical concepts. The search was kept as broad as possible by combining the Population (traumatic and nontraumatic SCI) and Outcomes (accidental falls) concepts. Minor publication types such as conference abstracts, letters, etc. were removed when possible such as from the Medline and Embase databases. The results were limited to humans. No date or language limits were applied. The full Medline search strategy is available for viewing in the Appendix.

Prior to abstract screening, the search results were deduplicated using reference management software (EndNote, Clarivate Analytics, Philadelphia, PA, USA). The abstracts were independently reviewed by two authors (ML and NU) to select articles for full-text review. Disagreements about the inclusion of an abstract were resolved by having a third author (KEM) review the abstract and make a decision. The inclusion and exclusion criteria for abstract and full-text screening were determined a priori. The inclusion criteria were: (1) study participants had a SCI acquired after birth; (2) study participants were 18 years or older; (3) study must have reported a measure of the occurrence of falls (e.g., proportion of participants who fell, number of falls/participant), or reported factors associated with falls in the participants. The exclusion criteria included: (1) review papers with no original data presented; (2) animal studies; 3) study participants with congenital SCI (i.e., spina bifida); (4) studies involving participants with a variety of health conditions, if SCI-specific data could not be extracted; and (5) studies examining falls solely as a cause of SCI.

\section{Data extraction}

Following full-text screening, two authors (AK and CP) independently extracted data from the included articles into a data extraction table. Any discrepancies between the two authors in the extracted data were resolved by having a third reviewer (KEM) read the article and extract the data in question. The data extracted are listed in Table 1. 
Table 1 Data extraction

\begin{tabular}{l}
\hline Data extracted from included articles \\
\hline -Study design \\
- Number of participants \\
- Characteristics of the participants \\
${ }^{\circ}$ Mean age \\
${ }^{\circ}$ Neurological level of injury \\
${ }^{\circ}$ American Spinal Injury Association Impairment Scale (AIS) \\
classification \\
${ }^{\circ}$ Cause of SCI (trauma, non-trauma, or both) \\
-Inclusion and exclusion criteria of the study \\
- Recruitment method(s) used \\
-Dates during which the study was conducted \\
• Retrospective (i.e., asking participants about falls in the past) or \\
prospective (i.e. tracking falls over time) data collection \\
-Method of data collection (e.g., survey, interview) \\
•Length of data collection (e.g., 6 months, 12 months) \\
- Study setting (i.e., inpatient/hospital or community) \\
•Definition of a fall \\
• Reported frequency of falls (or similar measure) \\
• Factors found to be associated with falls \\
• Consequences of the falls (e.g., injuries, psychosocial impact)
\end{tabular}

\section{Data synthesis}

To evaluate the risk of bias in the included articles (i.e., the likelihood the findings deviated from the truth), a domainbased approach was used [14]. This approach is a framework for evaluating the risk of bias based on aspects of the study design. The likelihood of bias for each domain is rated as low, high or unclear. Two domains were evaluated: (1) Measurement bias, which reflected the method(s) used to collect data on falls (i.e., retrospective methods, which are prone to recall bias [15], or prospective methods); and (2) Sampling bias, which considered how representative the study participants were of the larger SCI population. Sampling bias was evaluated by considering the recruitment method(s) used to enroll participants in the study. The recruitment strategy used in each study was classified according to the following four categories [16] (listed from high to low threat of bias, excluding the "Not stated" category):

- Convenience: prticipants were recruited from a single hospital or health center.

- Community-based: participants were recruited from more than one hospital or health center with the aim of reaching all eligible individuals in a specific geographical area (e.g., town, city).

- Population-based: participants were recruited from multiple hospitals or centers, as per community-based, but covered a larger geographical area (e.g., state/ province or country).

- Not stated: no information on the recruitment method was provided.

Whenever possible, the proportion of the study sample that experienced at least one fall during the study period was reported (i.e., incidence proportion) [17]. The incidence proportion of fallers is reported with a 95\% confidence interval (CI) that was calculated using Jeffrey's method [18]. The included articles were grouped according to the following categories in order to compare the incidence proportion and contributors to falls by: (1) stage of recovery (i.e., inpatient/hospital-dwelling participants versus community-dwelling participants); and (2) mobility status (i.e., ambulators versus wheelchair users). A random-effects meta-analysis [17] was used to pool the incidence proportion of the sub-groups when this metric was reported in two or more included articles [19]. $I^{2}$ and Q statistics assessed heterogeneity. If heterogeneity was large, a leave-one-out analysis was completed to evaluate each study's influence on the summary proportion incidence and $I^{2}$ [20]. R v 3.5.2 (The R Foundation, Vienna, Austria) was used to complete the meta-analyses.

To summarize the findings concerning the factors contributing to falls, the identified factors were classified according to the Biological, Behavioral, Social and Economic, and Environmental (BBSE) Model of fall risk [21]. The BBSE Model was developed to illustrate the multifactorial nature of falls and provide a means to gauge an individual's, or a group's, risk of falls [22]. The greater the number of risk factors identified across the four domains, the greater the risk [22]. The identified factors were grouped into biological, behavioral, social and economic, and environmental domains by three authors (AK, CP, JD) through discussion.

\section{Results}

\section{Results of screening}

The systematic search returned 2668 articles; after deduplication the total number of articles was reduced to 1706. Following abstract screening, 33 articles qualified for full-text review, and 25 of these articles were included in the review (see Fig. 1). Two studies reported data from the same data set $[23,24]$ and were combined for reporting and all analyses. Another two studies [25, 26] had some overlap of participants (personal communication, S. Amatachaya), but as each study included some unique participants, these two studies were kept separate for reporting and analysis. Thus, 24 unique studies were included. All articles were 
Fig. 1 Preferred Reporting Items for Systematic Reviews and Meta-Analysis (PRISMA) flow diagram summarizing the identification, screening, inclusion, and exclusion of articles

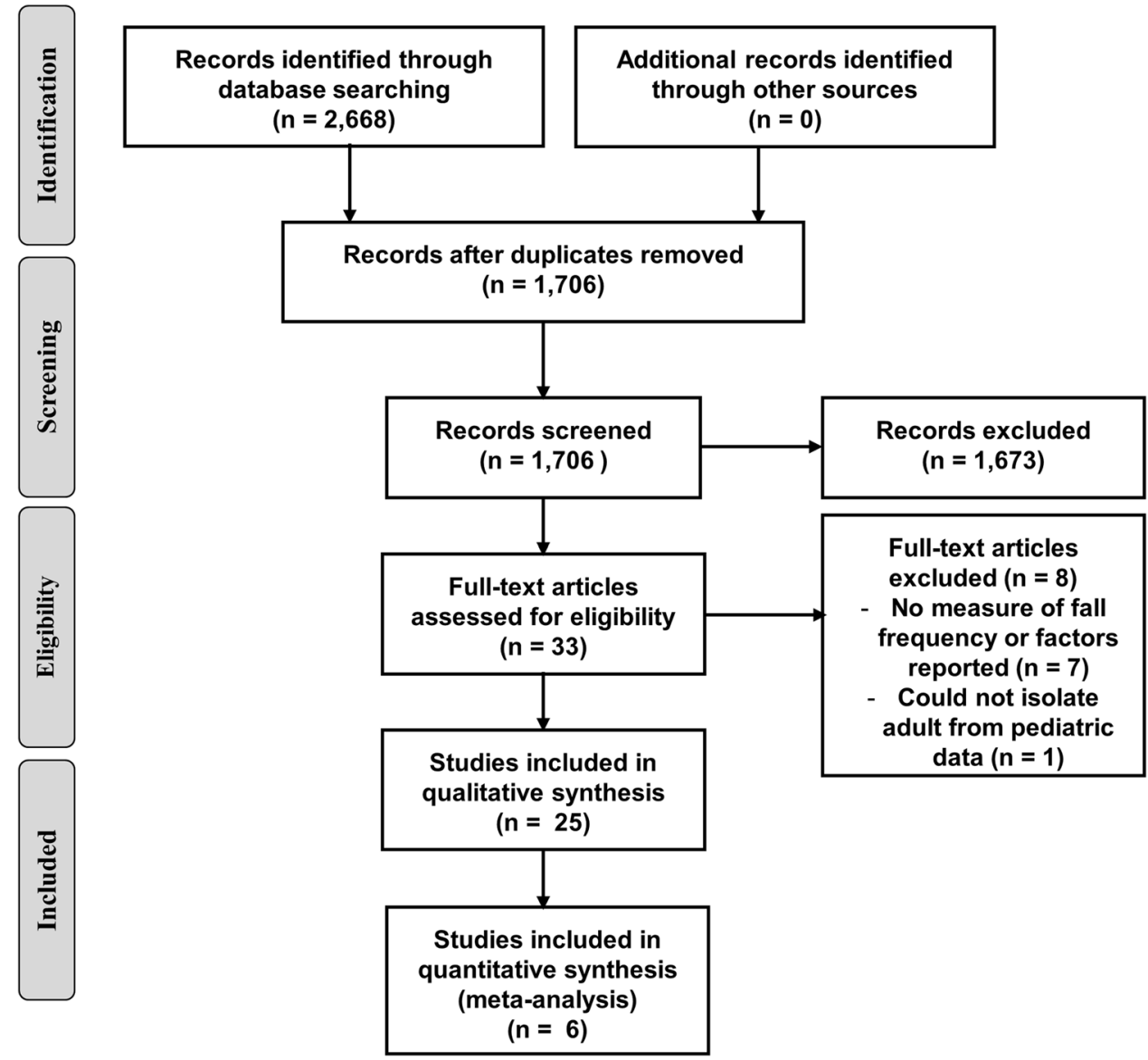

published between 2003 and 2017, with $17(68 \%)$ of the articles published in 2013 or later. All studies used quantitative research methods with the exception of the four most recently published articles, which used mixed methods [27] or qualitative methodologies [28-30] to learn about the experience of falling from study participants.

More than 3350 individuals with SCI participated in the 24 studies. The characteristics of the study participants varied across studies (see Supplementary Material 1). All but three studies $[8,27,31]$ included participants with SCI only. Males made up 35-83\% of the study sample, with the exception of two studies that sampled American veterans, resulting in samples comprised of $96 \%$ males [32, 33], and one qualitative study in which all seven participants were male [24]. The severity of SCI ranged from AIS A through $\mathrm{D}$, with some studies focusing on ambulatory individuals and others focused on wheelchair users. Only one study looked exclusively at falls during inpatient rehabilitation [31], whereas one study included individuals participating in inpatient and outpatient rehabilitation [34]. The remaining studies examined falls in community-dwelling individuals with SCI. See Table 2 for a summary of the demographic and injury-related variables from each study.

\section{Study quality}

The risk of bias in the included studies was increased by the sampling methods used (see Supplementary Material 2). Fourteen studies (71\%) used a sample of convenience to recruit participants, whereas four studies (17\%) recruited from the larger community [33, 35-37]. One study (4\%) recruited through national organizations for individuals with disabilities [8] and hence, adopted a population-based sampling approach. Two studies (8\%) did not specify the recruitment method used [27, 34].

Eleven studies $(46 \%)$ collected fall-related data retrospectively, increasing the potential for bias in the results. Retrospective methods included interviews [27, 34, 38, 39] or surveys $[8,23,24,35,37,40]$ about past falls history, and retrospective chart reviews [31, 32]. Twelve studies (50\%) prospectively collected fall-related data from the study participants. Prospective methods included weekly or monthly phone interviews $[25,26,33,36,41-44]$ or text messaging $[43,45]$ that in some studies was combined with a falls diary [25, 36, 42], and semi-structured interviews [28-30]. One study (4\%) collected data on falls both retrospectively (queried falls that occurred in the past month) 


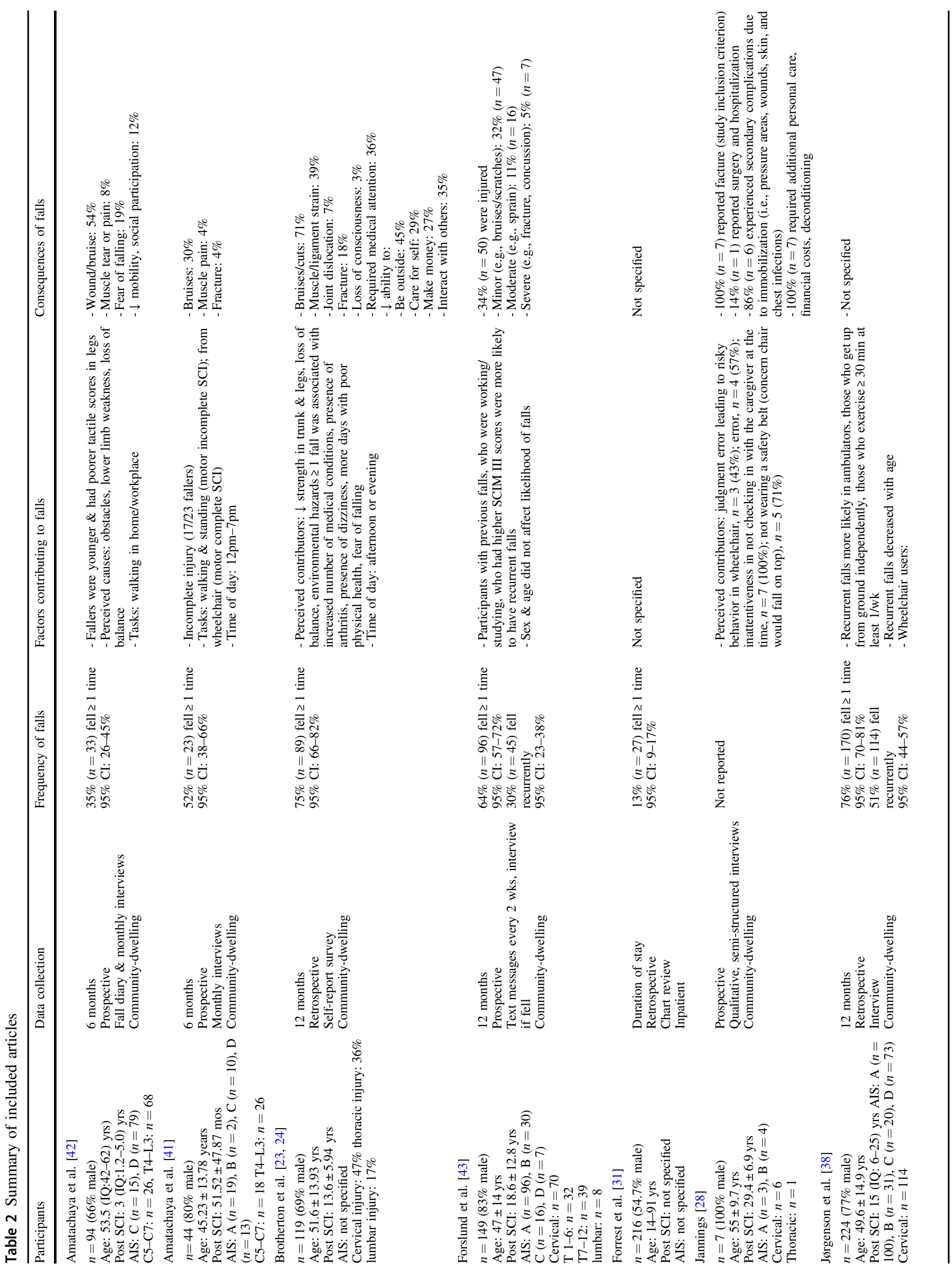




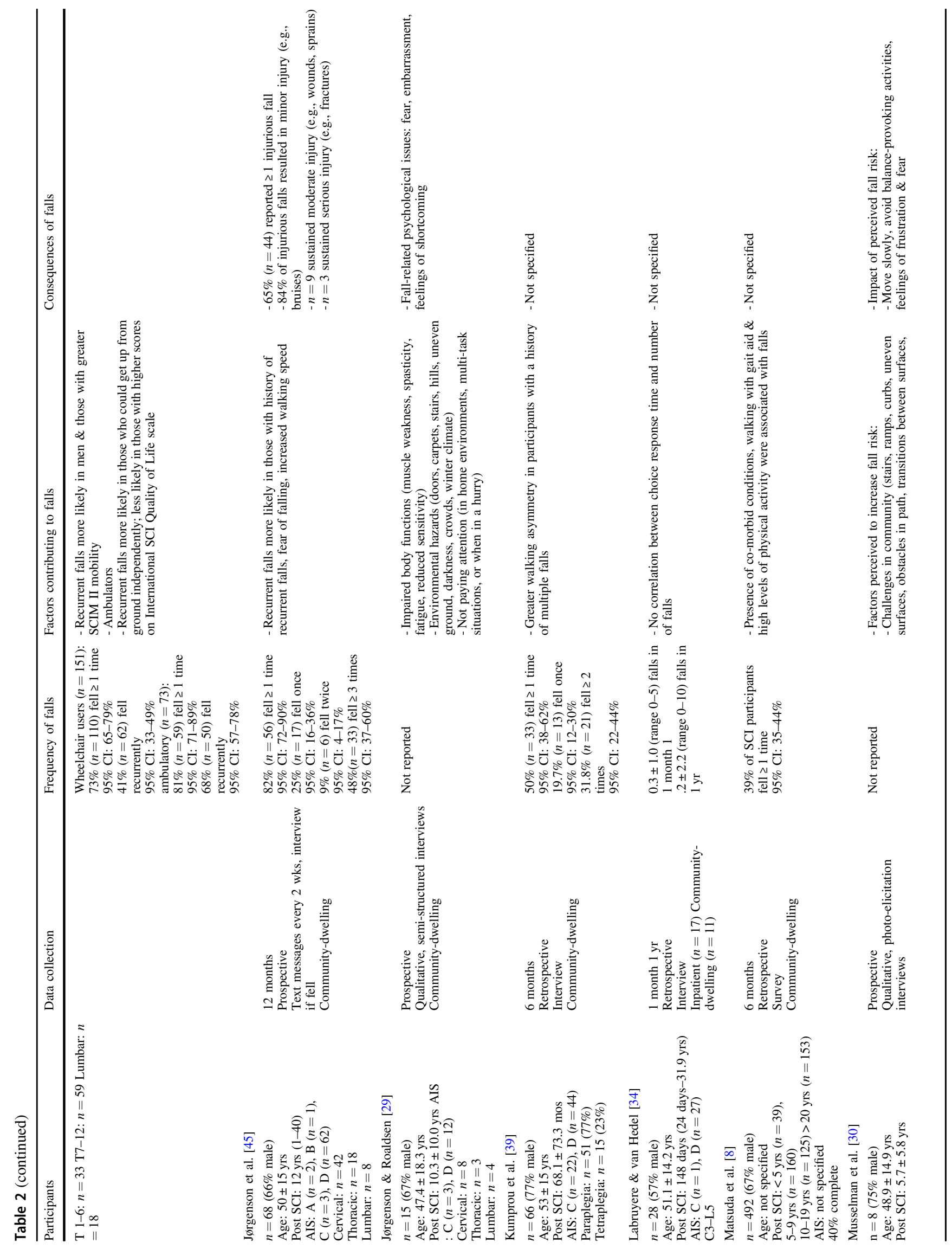




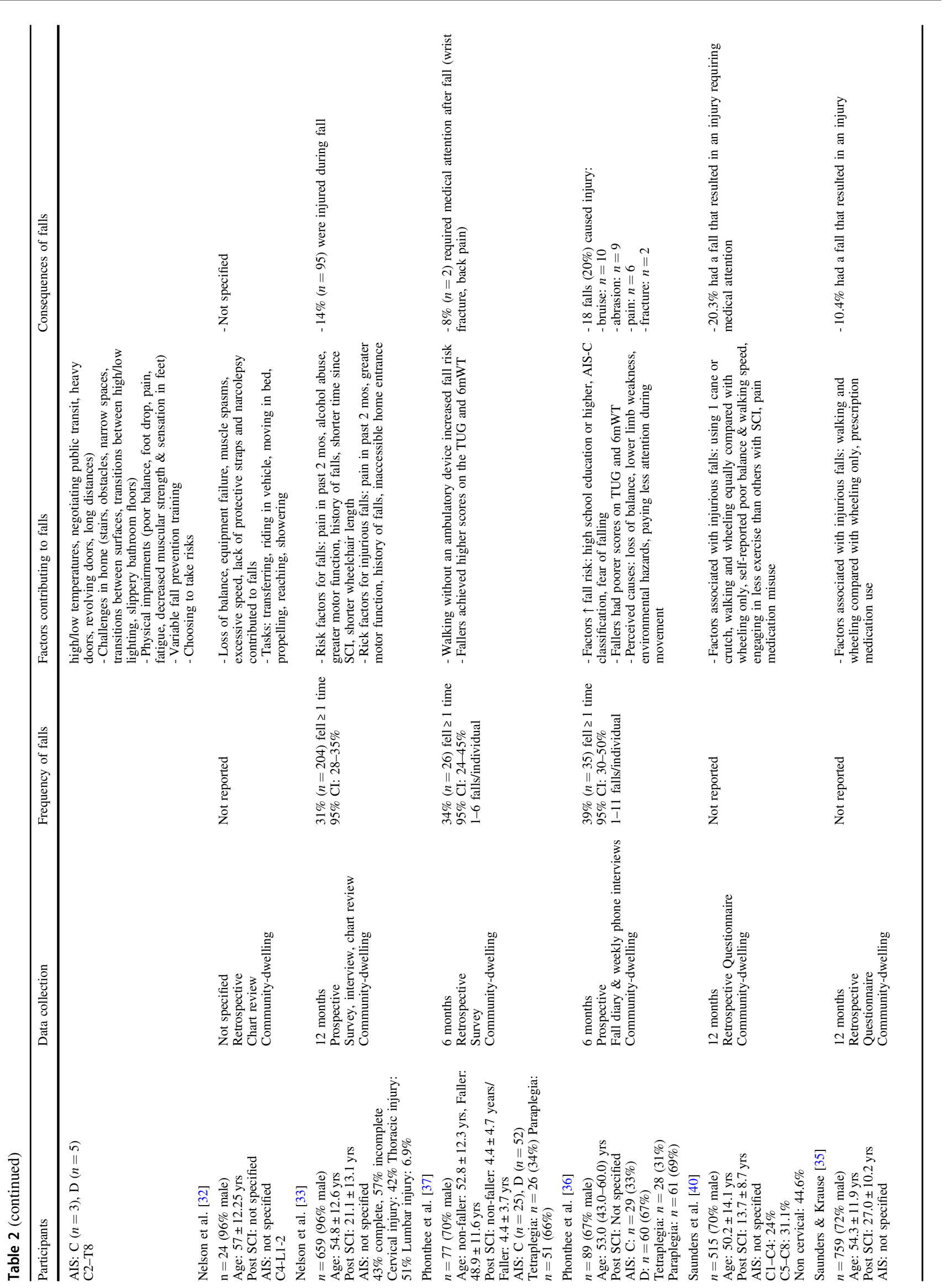




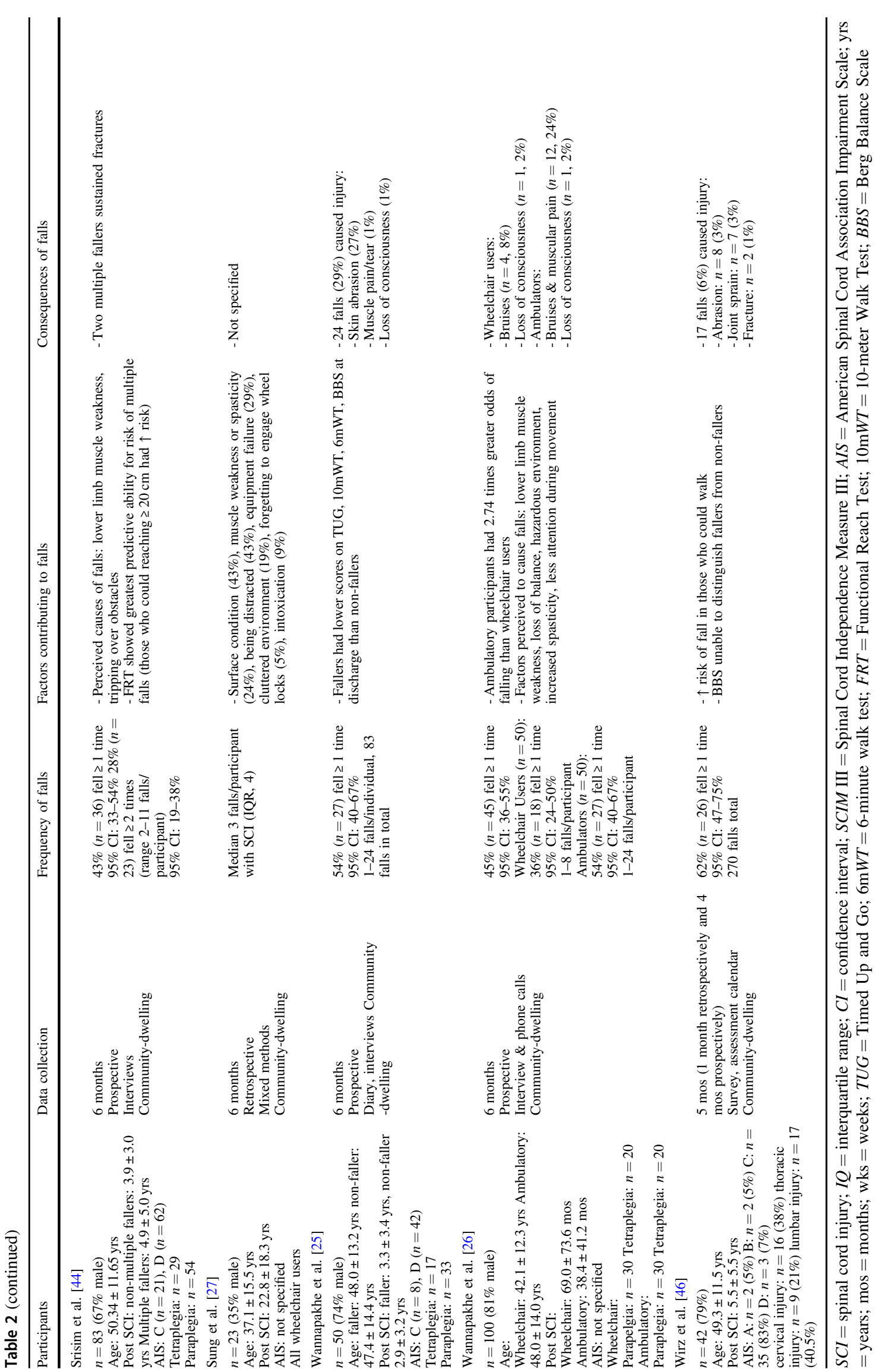


and prospectively (monitored falls over the subsequent four months using an "assessment calendar") [46].

\section{Frequency of falls}

Sixteen studies $(67 \%)$ provided a definition of a fall to guide their research. Similar descriptors were used to define falls across the included studies. Falls were described as unplanned and unexpected events where the individual contacted a lower support surface. Four studies (20\%) from the same research group required falls to occur while standing, walking or changing postures [36, 37, 42, 44].

The single study that sampled inpatients with SCI reported that $13 \%$ (95\% CI 9-17\%) of the sample experienced a fall during the rehabilitation hospital stay [31]. The duration of the inpatient stay, which varied from participant to participant, was not specified [31]. Among the remaining studies that examined falls in community-dwelling individuals, the length of data collection varied from 1 to 12 months. Among the studies that monitored falls prospectively, three collected data for 12 months [33, 43, 45], five collected data for 6 months [25, 26, 36, 41, 42, 44], and one for only 1 month [46]. Among studies that monitored falls retrospectively, four collected data for 12 months [23, $24,34,35,38,40]$, four for 6 months [8, 27, 37, 39], and one for 4 months [46].

Figure 2 is a synthesis of the proportion of communitydwelling study participants who fell at least once during the monitoring period of each study. Data are presented according to mobility status (ambulators and wheelchair users), length of data collection (six months and 12 months), and method of data collection (prospective and retrospective). The proportion of the study sample who fell ranged $34-82 \%$ for ambulatory participants and $31-73 \%$ for wheelchair users. The method of fall data collection did not appear to affect the reported incidence proportion of falls; however the length of data collection appeared to influence the metrics with a longer sampling period (i.e., 12 months) resulting in a larger proportion of fallers compare with a shorter sampling period (i.e., 6 months) (see Fig. 2). For this reason, meta-analyses were completed using data collected over 12 months only for ambulators and wheelchair users. The pooled incidence proportion of falls for ambulators was $78 \%$ (95\% CI 73-83\%) with little heterogeneity $\left(I^{2}=0 \%\right.$, $Q=1.79, p=0.41)$. The meta-analysis for wheelchair users initially revealed high heterogeneity $\left(I^{2}=98 \%, Q=112.27\right.$, $p<0.001)$ and a pooled incidence proportion of falls of $56 \%$ (95\% CI 28-81\%). When the study by Nelson et al. [33]. was removed from the meta-analysis, the heterogeneity was lower and non-significant $\left(I^{2}=59 \%, Q=2.46, p=0.12\right)$. Hence the pooled incidence proportion of falls for wheelchair users was calculated without Nelson et al. [33] and was found to be $69 \%$ (95\% CI 60-76\%).

The incidence proportion of study participants who experienced multiple falls (i.e., $>1$ fall) during the study period ranged $30-41 \%$ for wheelchair users [38, 43] and 28-68\% for ambulators [38, 39, 44, 45]. Jørgensen and colleagues [38] reported that the odds of recurrent falls were
Fig. 2 Percentage of study sample that experienced one or more falls. All studies shown here involved communitydwelling individuals with SCI. $\mathrm{WC}=$ wheelchair. 95\% Confidence intervals calculated using Jeffrey's method [18]

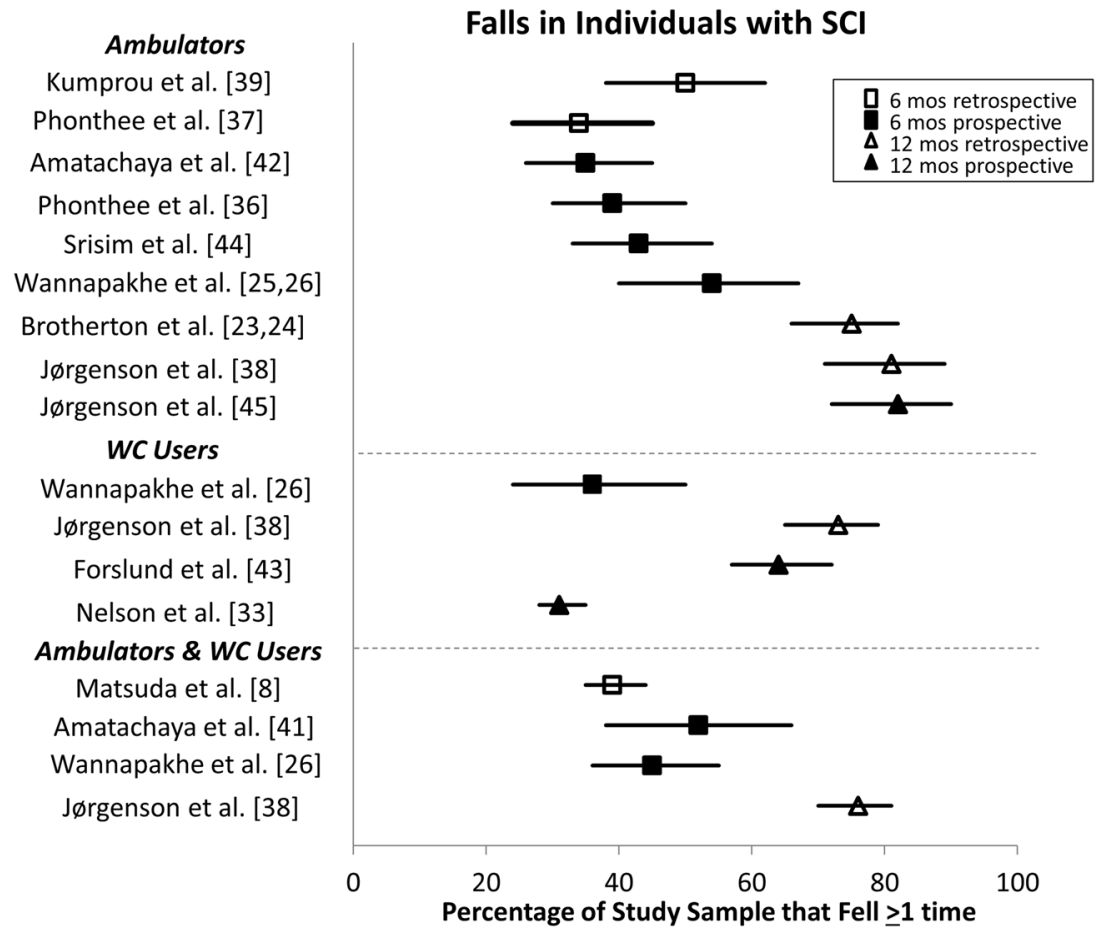


29 times higher for ambulators compared with wheelchair users.

\section{Factors contributing to falls}

When the factors identified as contributing to falls in community-dwelling individuals with SCI were organized according to the BBSE Model, numerous biological, behavioral, and environmental factors were reported (see Table 3 for factors and number of articles reporting each one). In terms of biological factors, loss of balance, muscular weakness, and spasticity or muscle spasms were commonly reported as contributors to falls. Level of function or mobility, as evidenced through performance on clinical measures (e.g., Spinal Cord Injury Independence Measure (SCIM) III, Timed Up and Go (TUG)) was also associated with the risk of falling. While some studies found a higher level of function or mobility to be associated with falls $[33,37,38,43,44]$ others found a lower level of function or mobility to be linked to an increased risk of falling [25, 36, 45]. Other biological factors reported by a few studies each included: a younger age, the presence of co-morbidities, trunk weakness, pain, fatigue, walking asymmetry, incomplete injury, male sex, narcolepsy, reduced sensation, and a shorter time since sustaining a SCI.

As for behavioral factors contributing to falls, inattention or distraction was identified most often, followed by ambulation, the presence of a fear of falling, a history of previous falls, and not using the safety features of the wheelchair or walking aid. Other behavioral factors identified by one or two studies were: risk-taking, engagement in physical activity, walking without a gait aid, walking with a gait aid, excessive speed, and alcohol consumption.

Numerous environmental factors, both within and outside of the home were identified (see Table 3). Hazards within the environment, without further detail about the specific hazards, were reported in two survey-based studies $[23,36]$. In contrast, two qualitative studies provided detailed information about structures and conditions within participants' environments that were perceived to increase the risk of falling, such as stairs, ramps/hills, heavy or revolving doors, and uneven or slippery surfaces [29, 30]. A few social and economic factors were identified: a higher level of education was associated with falls [32] as was not receiving adequate fall prevention training [30] and caregiver error [28].

The identified factors were subsequently examined for wheelchair users and for ambulators separately. Risk factors specific to the use of a wheelchair for mobility included driving with excessive speed [32], not using the chair's protective straps/safety belt/wheel locks [27, 28, 32], a shorter wheelchair length [33], and an inaccessible home entrance [33]. Male sex [38] and greater motor function
Table 3 Factors contributing to falls

\begin{tabular}{|c|c|}
\hline Biological & Behavioral \\
\hline $\begin{array}{l}\text { - Muscle weakness (8) } \\
\text { - Impaired balance (7) } \\
\text { - Greater function/mobility } \\
(5) \\
\text { - Muscle spasms/spasticity } \\
\quad(4) \\
\text { - Lower function/mobility } \\
\text { - R } \\
\text { - Reduced sensation (3) } \\
\text { - Greater number of co- } \\
\text { morbidities (2) } \\
\text { - Younger age (2) } \\
\text { - Pain (2) } \\
\text { - Fatigue (2) } \\
\text { - Trunk weakness (1) } \\
\text { - Walking asymmetry (1) } \\
\text { - Narcolepsy (1) } \\
\text { - Incomplete injury (1) } \\
\text { - Male sex (1) } \\
\text { - Shorter time since SCI (1) }\end{array}$ & $\begin{array}{l}\text { - Inattention/distraction during } \\
\text { movement (5) } \\
\text { - Ambulating (4) } \\
\text { - Fear of falling (3) } \\
\text { - Previous history of falls (3) } \\
\text { - Not using protective straps/safety } \\
\text { belt/wheel locks (3) } \\
\text { - Alcohol (2) } \\
\text { - Taking risks (2) } \\
\text { - Regular physical activity (2) } \\
\text { - Walk without gait aid (1) } \\
\text { - Walk with gait aid (1) } \\
\text { - Excessive speed (1) }\end{array}$ \\
\hline $\begin{array}{l}\text { Social \& economic } \\
\text { - High school education or } \\
\text { greater (1) } \\
\text { - Variable fall prevention } \\
\text { training (1) } \\
\text { - Caregiver error (1) }\end{array}$ & $\begin{array}{l}\text { Environmental } \\
\text { - Hazards in environment (4) } \\
\text { - Obstacles (3) } \\
\text { - Stairs (2) } \\
\text { - Uneven ground (2) } \\
\text { - Doors (2) } \\
\text { - Low lighting (2) } \\
\text { - Hills/ramps (2) } \\
\text { - Equipment failure (2) } \\
\text { - Curbs (1) } \\
\text { - Crowds (1) } \\
\text { - Carpet (1) } \\
\text { - Transition between surfaces (1) } \\
\text { - Slippery bathroom surfaces (1) } \\
\text { - Winter climate (1) } \\
\text { - High or low air temperature (1) } \\
\text { - Long distances to travel (1) } \\
\text { - Public transit (1) } \\
\text { - Narrow spaces in home (1) } \\
\text { - Inaccessible home entrance (1) } \\
\text { - Shorter length of wheelchair (1) }\end{array}$ \\
\hline
\end{tabular}

Classification of identified contributors to falls in individuals with SCI across all included studies. BBSE Model of Fall Risk [21] is used as the framework for classification. The number of studies reporting each contributor is indicated in parentheses after each contributor

${ }^{\mathrm{a}}$ Reported only in wheelchair users who fell

were additional factors identified in studies examining falls in wheelchair users. Specifically, higher SCIM II/III scores were associated with a greater risk of falling in wheelchair users [38, 43].

When considering the contributors to falls in ambulatory participants, contradictory findings were observed for the role of level of function and mobility. Two studies found that lower scores on the TUG and 6-minute walk test (6MWT) were associated with falls in ambulators [25, 36]. 
However, another study contrarily indicated that higher scores on these same tests (TUG, 6MWT) were contributors to falls [37], and Srisim and colleagues [44] reported that greater scores on the Functional Reach Test (i.e., an ability to reach $\geq 20 \mathrm{~cm}$ ) were associated with increased risk of falling. Similarly, walking both with [40] and without [37] a gait aid were identified as contributors to falls in separate studies. In terms of factors identified for ambulators, two studies found fear of falling to be a contributor $[23,36]$.

\section{The consequences of falls}

The reported proportion of falls that resulted in any injury ranged from 6 to $62 \%$ among ambulators and 13 to $38 \%$ among wheelchair users. The most common fall-related injuries among participants with SCI (ambulators and wheelchair users) were bruises and abrasions/cuts/scrapes. Other reported injuries included muscle tears or pain [42] and sprains, bloody nose, and numbness [33]. One study found that ambulators have a higher rate of minor injuries sustained through falling compared with wheelchair users [26]. Based on the four studies that looked at the percentage of fallers who sustained serious injuries requiring medical attention, reports ranged from 4 to $36 \%$ of participants [23, $24,41,43]$. The most commonly reported serious injuries included fracture and a loss of consciousness (see Table 2). In addition, one study, examining wheelchair users only, reported one death as a consequence of a fall, out of 204 fallers and 553 falls [33].

Regarding the psychosocial consequences of falls, increased fear of falling was reported by study participants [30, 42], as were feelings of embarrassment [29] and frustration [30]. Decreased participation in community activities was also reported as a consequence of falls by two studies [30, 42]. Likewise, social interaction was reported to decline as a result of falling in $12 \%$ [42] to $35 \%$ [23, 24] of fallers with incomplete SCI.

\section{Discussion}

We completed a systematic review and meta-analysis, following PRISMA guidelines, to summarize the incidence proportion of falls and factors contributing to falls in individuals with SCI. The majority of included studies used samples of convenience, thus increasing the potential for biased results. In this review we found that falling was common among community-dwelling individuals with SCI. While only one study reported on inpatient falls, we found a marked difference in the incidence proportion of falls in the hospital environment compared with the community. A variety of biological, behavioral and environmental factors contribute to falls and the increased risk of falling after SCI. The most commonly reported consequence of falls was a minor injury; however, up to $15 \%$ of falls result in a more serious injury, such as a fracture or loss of consciousness. Psychosocial consequences of falling also exist, such as feelings of fear, embarrassment and frustration, along with a reduced participation in daily activities. Altogether, this synthesis of the literature will assist with the development of SCI-specific fall prevention initiatives.

Individuals with SCI are at a high risk of falling, even when compared with other vulnerable populations. In a 12month period, $28-30 \%$ of able-bodied older adults [47, 48], $50 \%$ of individuals post-stroke [49] and $62-68 \%$ of individuals with Parkinson's disease $[50,51]$ will fall one or more times. Despite the frequent occurrence of falls among individuals with SCI, research concerning falls after SCI has only recently emerged; the majority of articles included in this review were published in the past 5 years. The increased study of falls among the SCI population, including both quantitative and qualitative methodologies, suggests increasing recognition of falls as a significant complication of SCI.

We found the reported yearly incidence proportion of falls to be similar between ambulators and wheelchair users with SCI. Prior to the recent publications by Forslund et al. [43]. and Jørgensen et al. [38]., the incidence proportion of falls among wheelchair users was reported to be lower (i.e. $<40 \%[26,33])$ than the current estimate of $69 \%$. Hence, evidence of high fall risk among wheelchair users is a relatively new finding.

This review identified only one study that quantified the number of falls in an inpatient hospital environment [31]; hence, the reported incidence proportion is not generalizable. This is a surprising observation given that the prevention of falls is an increasingly high priority for many hospitals, and greater emphasis has been placed on a safety culture and reducing patient harm during hospital stays [52, 53]. Our lack of knowledge concerning inpatient falls is a gap in our understanding of falls after SCI; the causes and circumstances of falls in an inpatient hospital environment may differ from those identified for community settings. The lower incidence proportion of falls among inpatients compared with community-dwelling individuals reported here may have resulted, in part, from the differing lengths of data collection. Although the length of inpatient stay was not reported in the study examining falls among inpatients [31], it is likely that the inpatient stays were shorter than the 6-12 months of monitoring in the community.

We identified $\sim 50$ factors that contribute to falls in individuals with SCI. However, this is likely not an exhaustive list as these findings are limited by the factors selected for study in the original articles. The BBSE model 
provided a useful framework for organizing this data and identifying the modifiable factors that may be targeted in fall prevention programs. The two most commonly identified biological factors, muscle weakness and impaired balance control, can be impacted through physical rehabilitation. A few behavioral factors, such as inattention/ distraction during movement and taking risks, could be targeted to increase awareness of these behaviors. Indeed, individuals with SCI have reported using strategies to reduce their risk of falling that involve modifying their behavior, such as increasing their awareness of their surroundings and modifying their movements to increase their stability [30]. Compared with biological and behavioral factors, environmental factors, especially those pertaining to community environments, may be more challenging to modify. It may be possible, however, to better prepare individuals with SCI to navigate challenging environments. Details concerning environmental factors related to falls were scarce until the recent publication of two qualitative studies [29, 30]. Detailed description of hazardous environments will assist clinicians in the design of appropriate simulated environments to help prepare individuals with SCI for safe mobility within their homes and communities.

The findings have led to several recommendations for future research. First, as mentioned above, there is a need to study the frequency and circumstances of falls during inpatient SCI rehabilitation as well as during the transition from hospital care to independent living in the community. Second, future research should include a combination of quantitative and qualitative methodologies to enable robust identification of fall-related trends while also targeting a detailed understanding of the nature and consequences of falls occurring among the SCI population. Third, researchers should be aware that the duration of monitoring will influence the incidence proportion of falls. As longitudinal studies lasting 12 months are not always feasible, we recommend a minimum monitoring period of 6 months, which would enable comparison of one's data with previously documented rates.

In conclusion, falls are a common event with physical and psychosocial consequences for community-dwelling individuals with SCI. The development of SCI-specific fall prevention initiatives, which aim to modify relevant biological and behavioral factors and to consider each individual's environmental risk, may result in a reduction of falls and safer mobility for the SCI population.

\section{Data archiving}

All extracted data can be found in Supplementary Material 1.
Acknowledgements We thank Katherine Chan, MSc, for assistance with data analysis.

Funding This research was funded by a Craig H. Neilsen Foundation grant (\#440070) to KEM.

Author contributions AK was responsible for data extraction and analysis, interpreting results and writing the manuscript. CP was responsible for data extraction and analysis, interpreting results and editing the manuscript. ML and NU were responsible for abstract and full-text screening and editing the manuscript. MP conducted the search and edited the manuscript. JD contributed to the interpretation of results and editing the manuscript. KEM was responsible for designing the review, overseeing screening and extraction, interpreting results and writing the manuscript.

\section{Compliance with ethical standards}

Conflict of interest The authors have no conflicts of interest to declare.

Publisher's note: Springer Nature remains neutral with regard to jurisdictional claims in published maps and institutional affiliations.

\section{References}

1. Scuffham P, Chaplin S, Leegood R. Incidence and costs of unintentional falls in older people in the United Kingdom. $\mathrm{J}$ Epidemiol. 2003;57:740-4.

2. World Health Organization. WHO Global Report on Falls Prevention in Older Age. 2007. Accessed on 9 July 2015. Available from: www.who.int/ageing/publications/Falls_prevention7March.pdf.

3. Veterans Affairs Canada. Seniors' falls - an emerging public health crisis. Accessed on 2 August 2017. Available from: https://www. veterans.gc.ca/eng/services/health/promotion/fallsp/senfalls.

4. Batchelor F, Hill K, Mackintosh S, Said C. What works in falls prevention after stroke?: a systematic review and meta-analysis. Stroke. 2010;41:1715-22.

5. Allen NE, Schwarzel AK, Canning CG. Recurrent falls in Parkinson's disease: a systematic review. Parkinson's Dis. 2013;2013:906274.

6. Canning CG, Paul SS, Nieuwboer A. Prevention of falls in Parkinson's disease: a review of fall risk factors and the role of physical interventions. Neurodegener Dis Manag. 2014;4:203-21.

7. Cameron M, Coote S, Sosnoff JJ. Whom to target for fallsprevention trials: recommendations from the international MS falls prevention research network. Int J MS Care. 2014;16:203-7.

8. Matsuda PN, Verrall AM, Finlayson ML, Molton IR, Jensen MP. Falls among adults aging with disability. Arch Phys Med Rehabil. 2015;96:464-71.

9. Craven BC, Verrier M, Balioussis C, et al. Rehabilitation Environmental Scan Atlas: Capturing Capacity in Canadian SCI Rehabilitation. 2012. Rick Hansen Institute, Canada. Accessed 13 October 2017. Available from: https://rickhanseninstitute.org/escan-atlas.

10. Skelton F, Hoffman JM, Reyes M, Burns SP. Examining healthcare utilization in the first year following spinal cord injury. J Spinal Cord Med. 2015;38:690-5.

11. Devivo MJ. Epidemiology of traumatic spinal cord injury: trends and future implications. Spinal Cord. 2012;50:365-72.

12. Thompson C, Mutch J, Parent S, Mac-Thiong J. The changing demographics of traumatic spinal cord injury: an 11-year study of 831 patients. J Spinal Cord Med. 2015;38:214-23. 
13. Moher D, Liberati A, Tetzlaff J, Altman DG. Preferred reporting items for systematic reviews and meta-analyses: the PRISMA statement. Ann Intern Med. 2009;151:264-9.

14. Higgins JP, Altman DG, Sterne JA. Assessing risk of bias in included studies. In: Higgins JP, Green S, editors. Cochrane handbook for systematic reviews of interventions. Version 5.1.0. The CochraneCollaboration; March 2011, chapter 8. Accessed May 23, 2016. Available at: http://www.cochranehandbook.org.

15. Rothman KJ, Greenland S, Lash TL. Case-control studies. In: Rothman KJ, Greenland S, Lash TL (eds). Modern Epidemiology. 3rd edn. (Lippincott Williams \& Wilkins, Philadelphia, PA, 2008) pp 112.

16. Arora T, Oates AR, Lynd K, Musselman KE. Current state of balance assessment during transferring, sitting, standing and walking activities for the spinal cord injured population: a systematic review. J Spinal Cord Med 2018; 5:1-14.

17. Greenland S, Rothman KJ. Measures of occurrence. In: Rothman KJ, Greenland S, Lash TL, (eds). Modern Epidemiology. 3rd edn. Philadelphia, PA: Lippincott Williams \& Wilkins; 2008. p. 33.

18. Brown LD, Cai TT, DasGupta A. Interval estimation for a binomial proportion. Stat Sci. 2001;16:101-33.

19. Valentine JC, Pigott TD, Rothstein HR. How many studies do you need? A primer on statistical power for meta-analysis. J Educ Behav Stat. 2010;35:215-47.

20. Wang, N How to Conduct a Meta-Analysis of Proportions in R: A Comprehensive Tutorial. 2018. Accessed 17 January 2019. Available from: https://doi.org/10.13140/RG.2.2.27199.00161.

21. Canadian Patient Safety Institute. Reducing Falls and Injuries From Falls: Getting Started Kit. June 2013. Accessed March 24, 2016. Available from: www.safehealthcarenow.ca.

22. Canadian Patient Safety Institute. What's new in falls best practices? July 2013. Accessed 5 July 2016. Available from: http://www.patientsafetyinstitute.ca/en/NewsAlerts/News/pages/w hats-new-in-falls-best-practices.aspx.

23. Brotherton SS, Krause JS, Nietert PJ. Falls in individuals with incomplete spinal cord injury. Spinal Cord. 2007;45:37-40.

24. Brotherton SS, Krause JS, Nietert PJ. A pilot study of factors associated with falls in individuals with incomplete spinal cord injury. J Spinal Cord Med. 2007;30:243-50.

25. Wannapakhe J, Arayawichanon P, Saengsuwan J, Amatachaya S. Changes of functional ability in patients with spinal cord injury with and without falls during 6 months after discharge. Phys Ther. 2014;94:675-81.

26. Wannapakhe J, Arrayawichanon P, Saengsuwan J, Amatachaya S. Medical complications and falls in patients with spinal cord injury during the immediate phase after completing a rehabilitation program. J Spinal Cord Med. 2015;38:84-90.

27. Sung J, Trace Y, Peterson EW, Sosnoff JJ, Rice LA. Falls among full-time wheelchair users with spinal cord injury and multiple sclerosis: a comparison of characteristics of fallers and circumstances of falls. Disabil Rehabil. 2017;41:1-7. E-pub Oct 25.

28. Jannings W. A quality improvement project to investigate the circumstances of lower limb fractures in non-ambulant persons with spinal cord injury. JARNA. 2017;20:14-18.

29. Jørgensen V, Roaldsen KS. Negotiating identity and self-image: perceptions of falls in ambulatory individuals with spinal cord injury - a qualitative study. Clin Rehabil. 2017;31:544-54.

30. Musselman KE, Arnold C, Pujol C, Lynd K, Oosman S. Falls, mobility, and physical activity after spinal cord injury: an exploratory study using photo-elicitation interviewing. Spinal Cord Ser Cases. 2018;4:39.

31. Forrest G, Huss S, Patel V, Jeffries J, Myers D, Barber C, et al. Falls on an inpatient rehabilitation unit: risk assessment and prevention. Rehabil Nurs. 2012;37:56-61.

32. Nelson A, Ahmed S, Harrow J, Fitzgerald S, Sanchez-Anguiano A, Gavin-Dreschnack D. Fall-related fractures in persons with spinal cord impairment: a descriptive analysis. SCI Nurs. 2003;20:30-37.

33. Nelson AL, Groer S, Palacios P, Mitchell D, Sabharwal S, Kirby $\mathrm{RL}$, et al. Wheelchair-related falls in veterans with spinal cord injury residing in the community: a prospective cohort study. Arch Phys Med Rehabil. 2010;91:1166-73.

34. Labruyère R, van Hedel HJ. Instrument validity and reliability of a choice response time test for subjects with incomplete spinal cord injury: relationship with function. Arch Phys Med Rehabil. 2011;92:1443-9.

35. Saunders LL, Krause JS. Injuries and falls in an aging cohort with spinal cord injury: SCI aging study. Top Spinal Cord Inj Rehabil. 2015;21:201-7.

36. Phonthee S, Saengsuwan J, Siritaratiwat W, Amatachaya S. Incidence and factors associated with falls in independent ambulatory individuals with spinal cord injury: a 6-month prospective study. Phys Ther. 2013;93:1061-72.

37. Phonthee S, Saengsuwan J, Amatachaya S. Falls in independent ambulatory patients with spinal cord injury: incidence, associated factors and levels of ability. Spinal Cord. 2013;51:365-8.

38. Jørgensen V, Forslund EB, Franzén E, Opheim A, Seiger Å, Ståhle A, et al. Factors associated with recurrent falls in individuals with traumatic spinal cord injury: a multicenter study. Arch Phys Med Rehabil. 2016;97:1908-16.

39. Kumprou M, Amatachaya P, Sooknuan T, Thaweewannakij T, Amatachaya S. Is walking symmetry important for ambulatory patients with spinal cord injury? Disabil Rehabil. 2018;40:836-41.

40. Saunders L, DiPiro N, Krause J, Brotherton S, Kraft S. Risk of fall-related injuries among ambulatory participants with spinal cord injury. Top Spinal Cord Inj Rehabil. 2013;19:259-66.

41. Amatachaya S, Wannapakhe J, Arrayawichanon P, Siritarathiwat W, Wattanapun P. Functional abilities, incidences of complications and falls of patients with spinal cord injury 6 months after discharge. Spinal Cord. 2011;49:520-4.

42. Amatachaya S, Pramodhyakul W, Wattanapan P, Eungpinichpong W. Ability of obstacle crossing is not associated with falls in independent ambulatory patients with spinal cord injury. Spinal Cord. 2015;53:598-603.

43. Forslund EB, Jørgensen V, Franzén E, Opheim A, Seiger Å, Ståhle A, et al. High incidence of falls and fall-related injuries in wheelchair users with spinal cord injury: a prospective study of risk indicators. J Rehabil Med. 2017;49:144-51.

44. Srisim K, Saengsuwan J, Amatachaya S. Functional assessments for predicting a risk of multiple falls in independent ambulatory patients with spinal cord injury. J Spinal Cord Med. 2015;38:439-45.

45. Jørgensen V, Forslund EB, Opheim A, Franzén E, Wahman K, Hultling $\mathrm{C}$, et al. Falls and fear of falling predict future falls and related injuries in ambulatory individuals with spinal cord injury: a longitudinal observational study. J Physiother. 2017;63:108-13.

46. Wirz M, Müller R, Bastiaenen C. Falls in persons with spinal cord injury: validity and reliability of the Berg Balance Scale. Neurorehabil Neural Repair. 2010;24:70-77.

47. Prudham D, Evans JG. Factors associated with falls in the elderly: a community study. Age Ageing. 1981;10:141-6.

48. Public Health Agency of Canada. Seniors' Falls in Canada. Second Report. 2014. Accessed 9 July 2016. Available at: http://www.phac-aspc.gc.ca/seniors-aines/publications/public/ injury-blessure/seniors_falls-chutes_aines/assets/pdf/seniors_fallschutes_aines-eng.pdf.

49. Simpson LA, Miller WC, Eng JJ. Effect of stroke on fall rate, location and predictors: a prospective comparison of older adults with and without stroke. PLoS ONE. 2011;6:e19431.

50. Stack E, Ashburn A. Fall events described by people with Parkinson's disease: implications for clinical interviewing and the research agenda. Physiother Res Int. 1999;4:190-200. 
51. Wood BH, Bilclough JA, Bowron A, Walker RW. Incidence and prediction of falls in Parkinson's disease: a prospective multidisciplinary study. J Neurol Neurosurg Psychiatry. 2002;72:721-5.

52. Spoelstra SL, Given BA, Given CW. Fall prevention in hospitals: an integrative review. Clin Nurs Res. 2012;21:92-112.
53. Singh H, Craven BC, Flett HM, Kerry C, Jaglal SB, Silver MP, et al. Factors influencing fall prevention for patients with spinal cord injury from the perspectives of administrators in Canadian rehabilitation hospitals. 57th Annual Meeting of the International Spinal Cord Society. Sydney, Australia. 13-15 September, 2018. 